NASA/CR-2003-212682

NIA Report No. 2003-05
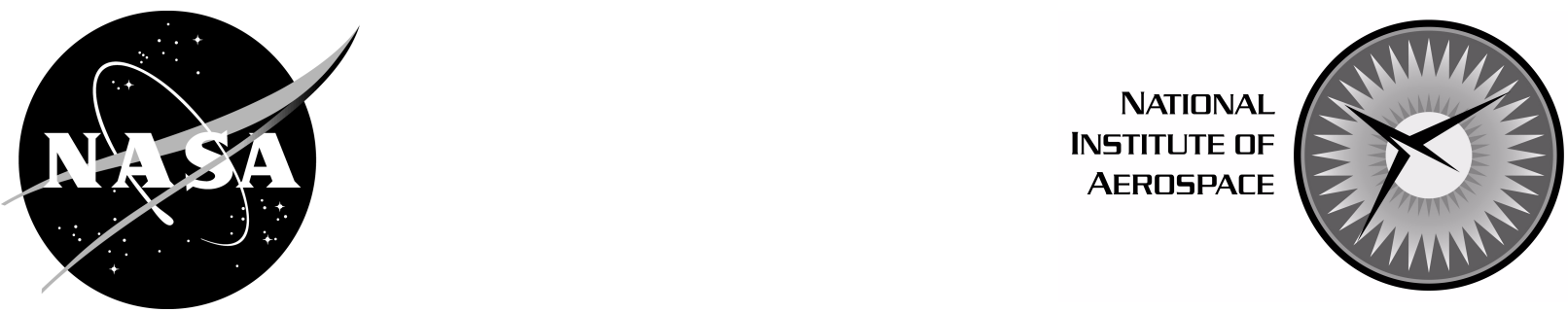

\title{
Parameter Transient Behavior Analysis on Fault Tolerant Control System
}

Jong-Yeob Shin

National Institute of Aerospace, Hampton, Virginia 


\section{The NASA STI Program Office . . . in Profile}

Since its founding, NASA has been dedicated to the advancement of aeronautics and space science. The NASA Scientific and Technical Information (STI) Program Office plays a key part in helping NASA maintain this important role.

The NASA STI Program Office is operated by Langley Research Center, the lead center for NASA's scientific and technical information. The NASA STI Program Office provides access to the NASA STI Database, the largest collection of aeronautical and space science STI in the world. The Program Office is also NASA's institutional mechanism for disseminating the results of its research and development activities. These results are published by NASA in the NASA STI Report Series, which includes the following report types:

- TECHNICAL PUBLICATION. Reports of completed research or a major significant phase of research that present the results of NASA programs and include extensive data or theoretical analysis. Includes compilations of significant scientific and technical data and information deemed to be of continuing reference value. NASA counterpart of peer-reviewed formal professional papers, but having less stringent limitations on manuscript length and extent of graphic presentations.

\section{- TECHNICAL MEMORANDUM.}

Scientific and technical findings that are preliminary or of specialized interest, e.g., quick release reports, working papers, and bibliographies that contain minimal annotation. Does not contain extensive analysis.

- CONTRACTOR REPORT. Scientific and technical findings by NASA-sponsored contractors and grantees.
- CONFERENCE PUBLICATION. Collected papers from scientific and technical conferences, symposia, seminars, or other meetings sponsored or co-sponsored by NASA.

- SPECIAL PUBLICATION. Scientific, technical, or historical information from NASA programs, projects, and missions, often concerned with subjects having substantial public interest.

- TECHNICAL TRANSLATION. Englishlanguage translations of foreign scientific and technical material pertinent to NASA's mission.

Specialized services that complement the STI Program Office's diverse offerings include creating custom thesauri, building customized databases, organizing and publishing research results ... even providing videos.

For more information about the NASA STI Program Office, see the following:

- Access the NASA STI Program Home Page at http://www.sti.nasa.gov

- Email your question via the Internet to help@sti.nasa.gov

- Fax your question to the NASA STI Help Desk at (301) 621-0134

- Telephone the NASA STI Help Desk at (301) 621-0390

- Write to: NASA STI Help Desk NASA Center for AeroSpace Information 7121 Standard Drive Hanover, MD 21076-1320 
NASA/CR-2003-212682

NIA Report No. 2003-05
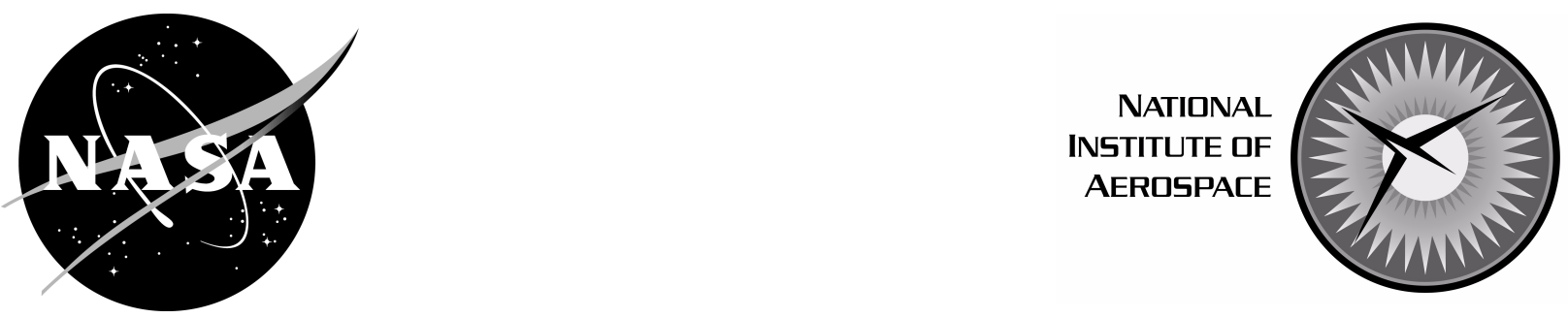

\section{Parameter Transient Behavior Analysis on Fault Tolerant Control System}

Jong-Yeob Shin

National Institute of Aerospace, Hampton, Virginia

National Aeronautics and

Space Administration

Langley Research Center

Hampton, Virginia 23681-2199
Prepared for Langley Research Center under Contract NCC-1-02043 
Available from the following:

NASA Center for AeroSpace Information (CASI)

7121 Standard Drive

Hanover, MD 21076-1320

(301) 621-0390
National Technical Information Service (NTIS)

5285 Port Royal Road

Springfield, VA 22161-2171

(703) 487-4650 


\title{
PARAMETER TRANSIENT BEHAVIOR ANALYSIS ON FAULT TOLERANT CONTROL SYSTEM*
}

\author{
Jong-Yeob Shin ${ }^{\dagger}$
}

\begin{abstract}
In a fault tolerant control (FTC) system, a parameter varying FTC law is reconfigured based on fault parameters estimated by fault detection and isolation (FDI) modules. FDI modules require some time to detect fault occurrences in aero-vehicle dynamics. This paper illustrates analysis of a FTC system based on estimated fault parameter transient behavior which may include false fault detections during a short time interval. Using Lyapunov function analysis, the upper bound of an induced- $\mathcal{L}_{2}$ norm of the FTC system performance is calculated as a function of a fault detection time and the exponential decay rate of the Lyapunov function.
\end{abstract}

\section{INTRODUCTION}

In the past decades, there has been interest in a FTC system which has ability to detect actuator/sensor faults automatically, and to prevent faults from developing into system failure. Especially, in designing a flight control system, it has been researched for achieving single aircraft accident prevention $[1,2,3,4,5]$. An active FTC system requires its control law to react to actuator/sensor faults through reconfiguration and fault detection and isolation (FDI) modules to detect actuator/sensor fault occurrences. FTC law synthesis for aerospace vehicles has been studied since 1980's based on possible faults on actuators and sensors [2]. Recently, using linear matrix inequality (LMI) optimization solutions [3, 4, 5], linear parameter varying (LPV) FTC laws are designed, based on an open-loop system modeled as a function of fault parameters. In the LPV-FTC synthesis procedure, it is assumed to be imminently identified by separate fault detection and isolation (FDI) modules. FDI modules are designed, based on only an open-loop system and applied in a closed-loop system $[5,6,7]$. In general, FDI modules and FTC laws are individually designed, without considering the other dynamics $[4,5]$.

After individually designing an LPV-FTC law and FDI modules, analysis of a FTC system is required to validate the FTC laws and FDI modules together. A typical way of analyzing a FTC system is nonlinear simulation with the pre-defined command inputs (not all possible command inputs), for possible fault scenario. It is hard to provide a generic criteria of a FTC system via nonlinear simulations. A FTC system analysis frame work is required to provide a certain criteria of a FTC system related with characteristics of FDI modules.

${ }^{*}$ This work was supported by the National Aeronautics and Space Administration under NASA Contract No. NAS1-02117.

${ }^{\dagger}$ Staff Scientist, National Institute of Aerospace (NIA), Hampton, VA 23666. Email: shinjy@nianet.org 
Usually, FDI modules can not detect faults at the moment a fault occurs. There is always some level of time delay to detect faults regardless of FDI algorithms such as an LPV-FDI filter [7] and an extended Kalman FDI filter [5]. During the delay time interval, the dynamics of the closed-loop system may not be in the pre-defined set of systems used in the LPV-FTC law synthesis procedure [5]. It is also possible that during the time interval, FDI modules may detect false faults which represent faults on healthy actuator/sensors. In this paper, the signal provided by FDI modules is parameterized and dynamics changes due to signal changes during the time interval are modeled as parameter transient behavior of an LPV system. It is possible that the parameter transient behavior includes that the system is locally unstable for a short time interval.

For that case, conventional LPV performance analysis methodologies can not be used since all systems are required to be locally stable for any fixed scheduling parameters. One of the LPV performance analysis methodologies with brief instabilities is developed in Ref. [8], using the main concept of switching system analysis. Modifying the analysis, in this paper, the upper bound of the induced- $\mathcal{L}_{2}$ norm of the closed-loop system is calculated in terms of possible delay time interval (a characteristic of FDI modules) and exponential decay rates of the Lyapunov function. A generic analysis framework of a FTC system is presented, including false fault parameter estimation effects on closed-loop system dynamics.

The analysis result of the proposed FTC system in this paper is one of indicators to find out which LPV-FTC law generates less performance degradation due to parameter transient behavior. In this paper, it is demonstrated by applying this FTC analysis frame work to a HiMAT FTC system designed in Ref. [5].

This paper contains the following sections. In section 2, a FTC system analysis problem is stated and an analysis methodology is described in section 3. In section 4, a HiMAT FTC system analysis is demonstrated. In section 5, this paper is concluded with a brief summary.

\section{PROBLEM STATEMENT}

In this section, analysis problems on a FTC system are described. The structure of a FTC system shown in Figure 1 is briefly described to carry out analysis problem objectives. A FTC system includes a FTC law, FDI modules and supervisory systems (logics). When a fault occurs, FDI modules and a supervisory system (logics) detect it and generate signals for evaluating/reconfiguring a FTC law. Assume that the designed FTC law can be represented as an LPV system or is designed using LPV synthesis methodologies in Ref. [9, 10, 5]. In this paper, FDI modules estimate fault parameters which can be converted into scheduling parameters of the designed FTC law via simple logics (see Fig. 1).

Fault parameters $\rho(t)$ represent actual fault occurrences on aero-vehicle dynamics $P(\rho(t))$. Assume that fault parameters $\rho(t)$ are in a piecewise continuous compact set $\mathcal{F}_{\rho} \subset \mathcal{R}^{n_{s}}$ to capture dynamics of abrupt fault occurrence. Since fault parameters are not directly measurable in real time via physical sensors, the parameters are estimated by FDI modules with estimation error bounds $\left(\delta_{\rho}\right)$. In practical terms, the size of $\delta_{\rho}$ is decided based on 
characteristics of FDI modules and possible fault scenarios. The designed LPV controller $K(\bar{\rho}(t))$ is evaluated based on the estimated fault parameters $\bar{\rho}(t)$ in a piecewise continuous compact set $\mathcal{F}_{\bar{\rho}} \subset \mathcal{R}^{n_{s}}$. Based on the LPV synthesis methodology [5], the designed LPV controller $K(\bar{\rho}(t))$ can robustly stabilize an aero-vehicle $P(\rho)$ under fault occurrences, when

$$
\rho(t) \in \mathcal{S}\left(\bar{\rho}, \delta_{\rho}\right):=\left\{\begin{array}{c}
\rho(t) \mid\|\rho(t)-\bar{\rho}(t)\| \leq \delta_{\rho}, \\
\rho \in \mathcal{F}_{\rho}, \bar{\rho} \in \mathcal{F}_{\bar{\rho}}, \quad 0 \leq \delta_{\rho}
\end{array}\right\}
$$

When a fault occurs, FDI modules require some time for estimated fault parameters to satisfy the condition: $\rho(t) \in \mathcal{S}\left(\bar{\rho}(t), \delta_{\rho}\right)$. The required time is called as detection time for FDI modules, hereafter. During the detection time interval, it is possible that fault parameters $\rho(t)$ are not in a set $\mathcal{S}\left(\bar{\rho}(t), \delta_{\rho}\right)$ since $\delta_{\rho}$ is generally chosen as smaller values than the entire range of $\rho(t)$ variation. The performance and stability for the closed-loop system is not guaranteed for the case: $\rho(t) \notin \mathcal{S}\left(\bar{\rho}(t), \delta_{\rho}\right)$ since the LPV control law is designed only for the case: $\rho(t) \in \mathcal{S}\left(\bar{\rho}(t), \delta_{\rho}\right)$. During the interval, parameter transient behavior[5] of the closed-loop system is observed for the case: $\rho(t) \notin \mathcal{S}\left(\bar{\rho}(t), \delta_{\rho}\right)$. When the time interval is long, the performance of the closed-loop system can be noticeably degraded and stability of the system can be also changed.

In order to analyze performance degradation and stability of the system, the closed-loop FTC system $G(\rho, \bar{\rho})$ is modeled as

$$
\begin{aligned}
\dot{x} & =A(\rho, \bar{\rho}) x+B(\rho, \bar{\rho}) d, \\
e & =C(\rho, \bar{\rho}) x+D(\rho, \bar{\rho}) d,
\end{aligned}
$$

where $x \in \mathcal{R}^{n_{x}}, d \in \mathcal{R}^{n_{d}}$ and $e \in \mathcal{R}^{n_{e}}$. All the matrices have compatible dimensions. Note that the fault parameters $\rho$ and estimated fault parameters $\bar{\rho}$ are treated as independent parameters in an analysis frame work.

The entire parameter space is defined as

$$
\mathcal{P}:=\left\{(\rho(t), \bar{\rho}(t)) \mid \rho(t) \in \mathcal{F}_{\rho}, \bar{\rho}(t) \in \mathcal{F}_{\bar{\rho}}\right\}
$$

and is divided into $m$ subspaces $\mathcal{P}_{i}$ such that

$$
\begin{aligned}
\mathcal{P} & =\bigcup_{i=1}^{m} \mathcal{P}_{i}:=\mathcal{P}_{1} \cup \mathcal{P}_{2} \cdots \cup \mathcal{P}_{m} \\
\emptyset & =\mathcal{P}_{i} \cap \mathcal{P}_{j}, \quad i \neq j, \quad i \in \mathcal{I}, \quad j \in \mathcal{I}, \\
& \mathcal{I}:=\{1,2, \cdots, m\} .
\end{aligned}
$$

Local and global stability of the closed-loop system $G(\rho, \bar{\rho})$ in Eq. (2) are defined over parameter subspaces as follows: 
Definition 1 Local and global stability:

Suppose there exists a positive definite matrix $P$ such that

$$
A^{T}(\rho, \bar{\rho}) P+P A(\rho, \bar{\rho})<0, \quad(\rho, \bar{\rho}) \in \mathcal{P}
$$

then the system is globally stable over the entire parameter set. When the condition in Eq. (5) is satisfied over only subsets $\mathcal{P}_{i}$, the system is locally stable.

To analyze system dynamics changes due to parameter transient behavior, it is necessary to introduce duration time of the system over each subspace.

Definition 2 Duration time $T_{p_{i}}$ over each subspace:

$$
\begin{gathered}
T_{p_{i}}\left(t_{o}, t\right)=\int_{t_{o}}^{t} \sigma_{i}(\rho(s), \bar{\rho}(s)) d s, \quad \forall t>t_{o}>0, \\
\sigma_{i}(\rho(s), \bar{\rho}(s))= \begin{cases}0, & (\rho(s), \bar{\rho}(s)) \notin \mathcal{P}_{i}, \\
1, & (\rho(s), \bar{\rho}(s)) \in \mathcal{P}_{i}, \\
& i \in \mathcal{I} .\end{cases}
\end{gathered}
$$

A duration time is bounded as

$$
T_{o_{i}}+\beta_{i}\left(t-t_{o}\right) \leq T_{p_{i}} \leq T_{o_{i}}+\alpha_{i}\left(t-t_{o}\right)
$$

where $0 \leq \beta_{i} \leq \alpha_{i} \leq 1$ and $0 \leq T_{o_{i}}$. The constant $\alpha_{i}$ represents a ratio of total time of interest to duration time over the $i$-th subspace. For example, when the system stays in the $i$-th subspace for all time, the constant $\alpha_{i}$ should be one. When the system never stays in the $i$-th subspace for all time, the constant $\alpha_{i}$ should be zero.

In this paper, we consider possible local instability over a subspace because FDI modules can generate false fault detection signals during the detection time interval. Without loss of generality, consider the case that the system is locally unstable in the $m$-th subspace. The constant $\alpha_{m}<1$ takes an important role in stability analysis to represent asymptotic stability ratio [8], which is related with stability margin of the system. In this paper, the constant $\alpha_{m}$ is interpreted as tolerance of the false detection of FDI modules for the closedloop system [11]. For example, if the system is stable with the larger number of $\alpha_{m}$, it implies that the system can stay in the $m$-th subspace longer.

In this paper, the subspace $\mathcal{P}_{1}$ is defined when $\rho(t) \in \mathcal{S}\left(\bar{\rho}(t), \delta_{\rho}\right)$. For practicality, assume that the closed-loop system stays in the subspace $\mathcal{P}_{1}$ for the most of the time of interest, since the FTC law is designed for it [5]. During the detection time interval, the closed-loop system stays in the parameter subspaces $\mathcal{P}_{i}, i \in \mathcal{I}-\{1\}$. The stability margin of the system over the entire parameter space can be described as a function of the constants $\alpha_{i}, i \in \mathcal{I}$. Also, the performance degradation level can be represented as an induced- $\mathcal{L}_{2}$ norm of the closed-loop system over the entire parameter space. 


\section{ANALYSIS METHOD}

In this section, the analysis problems described in the previous section are formulated into LMI forms.

\subsection{Stability Analysis}

Recall that the closed-loop system $G(\rho, \bar{\rho})$ in Eq. (2) is locally stable in the set of $\bigcup_{i=1}^{m-1} \mathcal{P}_{i}$ and locally unstable in the parameter subset $\mathcal{P}_{m}$.

Proposition 1: Suppose there exists a positive definite matrix $P(\bar{\rho})$ such that

$$
\begin{array}{ll}
A^{T}(\rho, \bar{\rho}) P+P A(\rho, \bar{\rho})+\dot{P} \leq-\lambda_{i} P, & (\rho, \bar{\rho}) \in \mathcal{P}_{i}, \quad i \in \mathcal{I}-\{m\}, \\
A^{T}(\rho, \bar{\rho}) P+P A(\rho, \bar{\rho})+\dot{P} \leq \kappa P, & (\rho, \quad \bar{\rho}) \in \mathcal{P}_{m},
\end{array}
$$

where

$$
0 \leq \kappa, \quad 0 \leq \lambda_{i} \leq \lambda_{1}, \quad i \in \mathcal{I}-\{1, m\}
$$

The system is exponentially stable with a decay rate:

$$
\lambda=\lambda_{1}-\sum_{i=2}^{m-1}\left(\lambda_{1}-\lambda_{i}\right) \alpha_{i}-\left(\lambda_{1}+\kappa\right) \alpha_{m}
$$

under the condition:

$$
\alpha_{m}<\alpha_{t}:=\frac{\lambda_{\min }}{\lambda_{\min }+\kappa},
$$

where $\lambda_{\min }=\min \left\{\lambda_{i}\right\}, i \in \mathcal{I}-\{m\}$.

The proof is follows. Set a Lyapunov function as $V=x^{T}(t) P(\bar{\rho}) x(t), \quad P \in \mathcal{R}^{n_{x} \times n_{x}}$. Using Eq. (9), the time derivative of the Lyapunov function is

$$
\begin{aligned}
& \dot{V} \leq-\lambda_{i} V,(\rho, \bar{\rho}) \in \mathcal{P}_{i}, \quad i \in \mathcal{I}-\{m\} \\
& \dot{V} \leq \kappa V, \quad(\rho, \quad \bar{\rho}) \in \mathcal{P}_{m} .
\end{aligned}
$$

From Eqs. (13) and (14),

$$
V \leq e^{-\lambda_{1}\left(t-t_{o}-\sum_{i=2}^{m-1} T_{p_{i}}\right)-\sum_{i=2}^{m-1} \lambda_{i} T_{p_{i}}+\kappa T_{p_{m}}} V\left(t_{o}\right), \quad \forall t \geq t_{o} \geq 0 .
$$

Taking the upper bound of the duration time over each parameter subspace, Eq. (15) is rewritten as:

$$
V \leq e^{-\lambda\left(t-t_{o}\right)+\sum_{i=2}^{m-1}\left(\lambda_{1}-\lambda_{i}\right) T_{o_{i}}+\left(\lambda_{1}+\kappa\right) T_{o_{m}}} V\left(t_{o}\right)
$$

where

$$
\lambda:=\lambda_{1}-\sum_{i=2}^{m-1}\left(\lambda_{1}-\lambda_{i}\right) \alpha_{i}-\left(\lambda_{1}+\kappa\right) \alpha_{m}
$$


Without loss of generality, the constant $\lambda_{1}$ can be set as $\max \left\{\lambda_{i}\right\}, i \in \mathcal{I}-\{m\}$. Thus, the term $\lambda_{1}-\lambda_{i}$ is always positive over the parameter subspaces $\mathcal{P}_{i}, i \in \mathcal{I}-\{1, m\}$. The lower bound of the constant $\lambda$ is

$$
\lambda \geq \lambda_{t}:=\lambda_{1}-\sum_{i=2}^{m-1}\left(\lambda_{1}-\lambda_{m i n}\right) \alpha_{i}-\left(\lambda_{1}+\kappa\right) \alpha_{m} .
$$

When the condition Eq. (12) is satisfied, it is easily noticed that the system is exponential stable such that $\lambda_{t} \geq 0$.

In this paper, the exponential decay rate is of interest to analyze a closed-loop system which may have local instability over a parameter subspace. The constant $\alpha_{t}$ represents the upper bound of asymptotic instability ratio [8]. In the analysis of a FTC system, the constant $\alpha_{t}$ can be interpreted as tolerance of instability during the detection time interval. For example, $\alpha_{t}=0.1$ implies that the closed-loop system can stay in the parameter subspace $\mathcal{P}_{m}$ for $10 \%$ of the total interest time without loss of exponential stability.

Given fault tolerant controllers, we can analyze the closed-loop system in terms of a constant $\alpha_{t}$ value. The stability analysis problem can be formulated into an optimization problem as:

$$
\begin{aligned}
& \max _{\kappa>0, \lambda_{\min }>0} \alpha_{t}, \\
& \text { s.t Eq. }(9)
\end{aligned}
$$

In this paper, the optimization is solved by checking feasibility of the LMI constraints in Eq. (9) with a line searching method over the constant $\lambda_{i}$ and $\kappa$ values.

\subsection{Performance analysis}

For the closed-loop system in Eq. (2), the induced- $\mathcal{L}_{2}$ norm is defined as:

$$
\sup _{(\rho, \bar{\rho}) \in \mathcal{P}, d \in \mathcal{L}_{2},\|d\|_{2} \neq 0} \frac{\|e\|_{2}}{\|d\|_{2}} .
$$

It is interesting to determine the induced $-\mathcal{L}_{2}$ norm from disturbance $d$ to error $e$ with parameter transient behavior during a detection time interval.

Proposition 2: Suppose there exists a positive definite matrix $P(\bar{\rho}) \in \mathcal{R}^{n_{x} \times n_{x}}$ such that

$$
\begin{aligned}
& (\rho, \bar{\rho}) \in \mathcal{P}_{i}, \quad i \in \mathcal{I}-\{m\}, \\
& {\left[\begin{array}{ccc}
A^{T}(\rho, \bar{\rho}) P(\bar{\rho})+P(\bar{\rho}) A(\rho, \bar{\rho})+\dot{P}(\bar{\rho})+\lambda_{i} P(\bar{\rho}) & P(\bar{\rho}) B(\rho, \bar{\rho}) & \gamma^{-1} C^{T}(\rho, \bar{\rho}) \\
B^{T}(\rho, \bar{\rho}) P(\bar{\rho}) & -I & \gamma^{-1} D^{T}(\bar{\rho}) \\
\gamma^{-1} C(\bar{\rho}) & \gamma^{-1} D(\bar{\rho}) & -I
\end{array}\right]<0,}
\end{aligned}
$$




$$
\begin{aligned}
& (\rho, \bar{\rho}) \in \mathcal{P}_{m}, \\
& {\left[\begin{array}{ccc}
A^{T}(\rho, \bar{\rho}) P(\bar{\rho})+P(\bar{\rho}) A(\rho, \bar{\rho})+\dot{P}(\bar{\rho})-\kappa P(\bar{\rho}) & P(\bar{\rho}) B(\rho, \bar{\rho}) & \gamma^{-1} C^{T}(\rho, \bar{\rho}) \\
B^{T}(\rho, \bar{\rho}) P(\bar{\rho}) & -I & \gamma^{-1} D^{T}(\rho, \bar{\rho}) \\
\gamma^{-1} C(\rho, \bar{\rho}) & \gamma^{-1} D(\rho, \bar{\rho}) & -I
\end{array}\right]<0 .}
\end{aligned}
$$

The induced- $\mathcal{L}_{2}$ norm from $d$ to e of the closed-loop system is no larger than $M_{\gamma}$ where

$$
\begin{aligned}
& M_{\gamma}=\gamma \sqrt{\frac{e^{\sum_{i=2}^{m-1}\left(\lambda_{1}-\lambda_{i}\right) T_{o_{i}}+\left(\lambda_{1}+\kappa\right) T_{o_{m}}} \lambda_{b}}{\lambda},} \\
& \lambda_{b}=\lambda_{1}-\sum_{i=2}^{m-1}\left(\lambda_{1}-\lambda_{i}\right) \beta_{i}-\left(\lambda_{1}+\kappa\right) \beta_{m}, \\
& \lambda \quad=\lambda_{1}-\sum_{i=2}^{m-1}\left(\lambda_{1}-\lambda_{i}\right) \alpha_{i}-\left(\lambda_{1}+\kappa\right) \alpha_{m} .
\end{aligned}
$$

Under the condition $\alpha_{m}<\alpha_{t}:=\frac{\lambda_{\min }}{\left(\lambda_{\min }+\kappa\right)}$, the constant $\lambda$ is always positive such that $\lambda \geq \lambda_{b}>0$.

The proof is follows. Set $V=x^{T}(t) P(\bar{\rho}) x(t)$, then the time derivative of a Lyapunov function $V$ is

$$
\begin{array}{ll}
\dot{V} \leq-\lambda_{i} V+\|d\|^{2}-\gamma^{-2}\|e\|^{2}, & (\rho, \bar{\rho}) \in \mathcal{P}_{i}, \quad i \in \mathcal{I}-\{m\} \\
\dot{V} \leq \kappa V+\|d\|^{2}-\gamma^{-2}\|e\|^{2}, & (\rho, \bar{\rho}) \in \mathcal{P}_{m} .
\end{array}
$$

Eqs. (21) and (22) describe the condition Eq. (24). The Lyapunov function is rewritten as:

$$
V(t) \leq e^{f\left(t_{0}, t\right)} V\left(t_{o}\right)+\int_{t_{o}}^{t} e^{f(s, t)}\left(\|d(s)\|^{2}-\gamma^{-2}\|e(s)\|^{2}\right) d s,
$$

where

$$
f(s, t)=-\lambda_{1}\left(t-s-\sum_{i=2}^{m} T_{p_{i}}(s, t)\right)-\sum_{i=2}^{m-1} \lambda_{i} T_{p_{i}}(s, t)+\kappa T_{p_{m}}(s, t) .
$$

Since $V(t)>0, \quad \forall t \geq t_{o} \geq 0$, the following inequality equation is extracted from Eq. (25).

$$
\gamma^{-2} \int_{t_{o}}^{t} e^{f(s, t)}\|e(s)\|^{2} d s \leq e^{f\left(t_{o}, t\right)} V\left(t_{o}\right) \quad+\int_{t_{o}}^{t} e^{f(s, t)}\|d(s)\|^{2} d s, \quad \forall t \geq t_{o} \geq 0 .
$$

Using Eq. (8) and the definition of $\lambda$ and $\lambda_{b}$ in Eq. (23), it is easily derived that

$$
\begin{aligned}
& \gamma^{-2} \int_{t_{o}}^{t} e^{-\lambda_{b}(t-s)}\|e(s)\|^{2} d s \leq e^{\sum_{i=2}^{m-1}\left(\lambda_{1}-\lambda_{i}\right) T_{o_{i}}+\left(\lambda_{1}+\kappa\right) T_{o_{m}}-\lambda\left(t-t_{o}\right)} V\left(t_{o}\right) \\
& +\int_{t_{o}}^{t} e^{\sum_{i=2}^{m-1}\left(\lambda_{1}-\lambda_{i}\right) T_{o_{i}}+\left(\lambda_{1}+\kappa\right) T_{o_{m}}-\lambda(t-s)}\|d(s)\|^{2} d s .
\end{aligned}
$$


Integrating both sides of Eq. (28) over the interval $\left(t_{o}, \infty\right)$ leads to

$$
\frac{\gamma^{-2}}{\lambda_{b}} \int_{t_{o}}^{\infty}\|e(s)\|^{2} d s \leq \frac{V\left(t_{o}\right)}{\lambda} e^{\sum_{i=2}^{m-1}\left(\lambda_{1}-\lambda_{i}\right) T_{o_{i}}+\left(\lambda_{1}+\kappa\right) T_{o}}+\frac{1}{\lambda} e^{\sum_{i=2}^{m-1}\left(\lambda_{1}-\lambda_{i}\right) T_{o_{i}}+\left(\lambda_{o}+\kappa\right) T_{o}} \int_{t_{o}}^{\infty}\|d(s)\|^{2} d s .
$$

Thus, the upper bound of the induced- $\mathcal{L}_{2}$ norm $M_{\gamma}$ is

$$
M_{\gamma}=\gamma \sqrt{\frac{e^{\sum_{i=2}^{m-1}\left(\lambda_{1}-\lambda_{i}\right) T_{o_{i}}+\left(\lambda_{1}+\kappa\right) T_{o_{m}}} \lambda_{b}}{\lambda}}
$$

The constant $M_{\gamma}$ represents the upper bounds of the worst-case performance level under parameter transient behavior which includes possible local instability. Thus, the performance analysis problem is formulated into an optimization problem:

$$
\begin{gathered}
\min _{\lambda_{i}>0, \kappa \geq 0} M_{\gamma}\left(\min _{P \in \mathcal{R}^{n_{x} \times n_{x}}} \gamma\right), \\
\text { s.t. Eq. }(21),(22) .
\end{gathered}
$$

The minimization $\gamma$ problem at fixed $\lambda_{i}$ and $\kappa$ is an LMI problem. However, the optimization problem in Eq. (31) is not an LMI problem in terms of $\lambda_{i}, \kappa$ and $\gamma$ with $P$. Also, the solution $\gamma$ is highly related with $\lambda_{i}$ and $\kappa$ values. In this paper, line searching over possible $\lambda_{i}$ and $\kappa$ ranges is used to find a solution of the optimization problem.

Note that this analysis does not require that the positive matrix $P$ is a function of the estimated parameters $\bar{\rho}$. Based on behaviour of estimated parameters by the FDI modules, the matrix $P$ can be set as a constant positive matrix or a function of parameter over the $\bar{\rho}$ variations. The constant positive matrix can allow abrupt change of the estimated parameter but leads to conservative analysis results. It will be demonstrated via the following example.

\section{EXAMPLE}

In this section, the proposed analysis methodology is demonstrated by application to a FTC system. Using the analysis, it is possible to indicate which FTC law is tolerant of possible false detection signal and generates less performance degradation for parameter transient behaviors during the detection time interval.

\subsection{FTC HiMAT System}

The FTC HiMAT system [5] consists of an on-line FDI system with simple logics, the dynamics of a HiMAT vehicle shown in Figure 2, and a designed LPV-FTC law. The HiMAT vehicle dynamics is taken from the $\mu$-synthesis Toolbox [12] and is modeled as an LPV system. The LPV model has two inputs: elevons $\delta_{e}$ and canards $\delta_{c}$; two outputs: angle of attack $\alpha$ in radians and pitch angle $\theta$ in radians; and four states: velocity $V$ in $\mathrm{ft} / \mathrm{sec}$, angle 
of attack $\alpha$, pitch rate $q$ in $\mathrm{rad} / \mathrm{sec}$, and pitch angle $\theta$. The LFT-LPV model of the HiMAT vehicle is

$$
\begin{gathered}
\dot{x}=A x+B\left[\begin{array}{cc}
\bar{\tau}_{1} & 0 \\
0 & \bar{\tau}_{2}
\end{array}\right] u+B\left[\begin{array}{cc}
0.1 & 0 \\
0 & 0.1
\end{array}\right] w, \\
z=u, \quad y=C x \\
w=\Delta z, \quad \Delta=\left[\begin{array}{cc}
\delta_{1} & 0 \\
0 & \delta_{2}
\end{array}\right]
\end{gathered}
$$

where the detailed elements of the system matrices $A, B$, and $C$ are in Ref. [5]. The estimated scheduling parameter $\bar{\rho}$ is defined using simple logics as

$$
\begin{array}{rll}
0 \leq \bar{\rho}<1 & : \quad 0 \leq \bar{\tau}_{1}<1, \bar{\tau}_{2}=1 \quad \text { Elevon failure } \\
\bar{\rho}=1 & : \quad \bar{\tau}_{1}=1, \quad \bar{\tau}_{2}=1 \\
1<\bar{\rho} \leq 2 & : \quad \bar{\tau}_{1}=1,0 \leq \bar{\tau}_{2}<1 \quad \text { Canard failure. }
\end{array}
$$

The on-line FDI model [5] estimates fault parameters $\bar{\tau}_{1}$ and $\bar{\tau}_{2}$ using the two-stage optimal Kalman filter $[5,13]$. Based on the estimated fault parameters, the estimated scheduling parameter $\bar{\rho}$ is defined via the simple logics.

LPV-FTC laws $K_{A}(\bar{\rho})$ and $K_{B}(\bar{\rho})$ are designed without a scaling factor using conventional LPV control synthesis methodology in Ref. [10] and with a scaling factor using a robust LPV synthesis methodology [5], based on the LPV-HiMAT model with estimated error bound 0.1. The closed-loop responses with the controllers $K_{A}$ and $K_{B}$ are simulated for the possible fault scenario of the canards failing at $1 \mathrm{sec}$ and are shown in Figure 3, respectively. The right plots in Figure 3 are magnifications of the left plots. Based on Figure 3, there is very little difference between $K_{A}$ and $K_{B}$ responses with steady state parameter estimates and pitch angle time responses. The simulation results in Figure 3 show that the detection time interval is about $0.5 \mathrm{sec}$ from $\mathrm{t}=1 \mathrm{sec}$ to $\mathrm{t}=1.5 \mathrm{sec}$. During the interval, a scheduling parameter $\rho$ is 2 , which indicates canard failure. The estimated parameter $\bar{\rho}$, however, is not 2 during the interval. It is easily observed that the condition $\rho(t) \in \mathcal{S}:=\{\rho|| \rho-\bar{\rho} \mid \leq 0.1\}$ is not satisfied during the interval. However, overall simulation results show that the closedloop system is stable and achieves the desired performance objective defined in Ref. [5] since the detection time interval is short in the simulation (less than $0.5 \mathrm{sec}$ ).

It is noticed, using linear analysis at fixed parameters, that the eigenvalues of the closedloop system with $K_{A}$ or $K_{B}$ are positive at fixed parameters $\bar{\rho}=0$ and $\rho=2$. When $\bar{\rho}>0.1$, the closed-loop system is stable at fixed parameters. This leads to the FTC HiMAT system being locally unstable for the short interval. 


\begin{tabular}{ccccc}
\hline \hline \multicolumn{5}{c}{ constant matrix $P$} \\
\hline Controller & $\lambda_{1}$ & $\lambda_{2}$ & $\kappa$ & $\alpha_{t}$ \\
\hline$K_{A}$ & 0.042 & 0.041 & 7.65 & $5.3 \times 10^{-3}$ \\
$K_{B}$ & 0.042 & 0.038 & 4.96 & $7.6 \times 10^{-3}$ \\
\hline \multicolumn{5}{c}{ parameter dependent $P(\bar{\rho})$} \\
\hline$K_{A}$ & 0.041 & 0.039 & 7.6 & $5.1 \times 10^{-3}$ \\
$K_{B}$ & 0.034 & 0.032 & 3.7 & $8.6 \times 10^{-3}$ \\
\hline \hline
\end{tabular}

Table 1: Stability analysis results

\subsection{Stability Analysis}

To apply the stability analysis methodology described in section 3 , the parameter set $\mathcal{P}$ is divided into three subspaces for this example. Set the subspaces as

$$
\begin{array}{ll}
\mathcal{P}_{1}=\{(\rho, \bar{\rho}) \mid \rho=2, & 0.9 \leq \bar{\rho} \leq 2\} \\
\mathcal{P}_{2}=\{(\rho, \bar{\rho}) \mid \rho=2, & 0.1 \leq \bar{\rho} \leq 0.9\} \\
\mathcal{P}_{3}=\{(\rho, \bar{\rho}) \mid \rho=2, & 0.0 \leq \bar{\rho} \leq 0.1\}
\end{array}
$$

Note that when $\bar{\rho} \in \mathcal{P}_{1}$, the condition $\rho(t) \in \mathcal{S}(\bar{\rho}, 0.1)$ is satisfied. Recall that the condition is used in designing an LPV-FTC law. When $\bar{\rho} \in \mathcal{P}_{2}$, the closed-loop system is locally stable but the condition is not satisfied. When $\bar{\rho} \in \mathcal{P}_{3}$, the closed-loop system is locally unstable.

The LMI constraints in Eq. (9) are evaluated at grid points $\bar{\rho} \in\{0,0.1,0.2, \cdots, 2\}$ over the parameter subspaces. In order to solve the optimization problem in Eq. (19) with LMI constraints in Eq. (9), the ranges of $\lambda_{1}, \lambda_{2}$, and $\kappa$ are defined as $0.01 \leq \lambda_{1} \leq 0.1$, $0.01 \leq \lambda_{2} \leq 0.1$, and $5 \leq \kappa \leq 10$, respectively. With fixed $\lambda_{1}, \lambda_{2}$, and $\kappa$ values in each range, the feasibility of the LMI constraints is checked with a constant matrix $P$ and a parameter dependent matrix $P(\bar{\rho})$, respectively. Using a parameter dependent matrix $P(\bar{\rho})$, the time derivative $\dot{\bar{\rho}}$ is required to determine $\dot{P}=\dot{\bar{\rho}} \frac{\partial P(\bar{\rho})}{\partial \bar{\rho}}$. In this example, it is assumed that $|\dot{\bar{\rho}}| \leq 1$, based on the FDI module characteristics. The parameter dependent matrix $P(\bar{\rho})$ is described using basis functions.

$$
P(\bar{\rho})=P_{o}+\bar{\rho} P_{1}+\frac{1}{\bar{\rho}} P_{2}, \quad \text { constant } P_{o, 1,2} \in \mathcal{R}^{n \times n},
$$

where $n$ is the closed-loop state order. The optimization results are in Table 1 . Recall that the constant $\alpha_{t}$ represents the ratio of possible duration time over the unstable parameter space to total time. Based on the calculated $\alpha_{t}$ values, both FTC laws have similar tolerance to possible false fault detection in the stability of the closed-loop system. When two controllers are compared, the controller $K_{B}$ has slightly better tolerance. It is also shown in Table 1 that 


\begin{tabular}{ccccc}
\hline \hline \multicolumn{5}{c}{ constant matrix $P$} \\
\hline Controller & $\lambda_{1}$ & $\lambda_{2}$ & $\kappa$ & $\gamma$ \\
\hline$K_{A}$ & $3 \times 10^{-3}$ & $1 \times 10^{-3}$ & 9.4 & 0.42 \\
$K_{B}$ & $6 \times 10^{-4}$ & $1 \times 10^{-4}$ & 5.85 & 2.14 \\
\hline \multicolumn{5}{c}{ parameter dependent $P(\bar{\rho})$} \\
\hline$K_{A}$ & $6 \times 10^{-4}$ & $1 \times 10^{-4}$ & 9.4 & 0.34 \\
$K_{B}$ & $6 \times 10^{-4}$ & $1 \times 10^{-4}$ & 3.7 & 0.63 \\
\hline \hline
\end{tabular}

Table 2: Performance analysis results

the analysis with parameter dependent $P$ leads to less conservative results on the controller $K_{B}$.

\subsection{Performance Analysis}

It is of interest to know how much performance of the closed-loop system degrades due to parameter transient behaviors. To analyze the closed-loop performance, the desired response function $T_{i}$ and the performance weighting function $W_{p}$ in Figure 4 are defined as

$$
T_{i}=\frac{1}{s / 0.8+1}, \quad W_{p}=40 \frac{s / 50+1}{s / 0.05+1} .
$$

Note that the weight functions are, also, used in an LPV control synthesis in Ref. [5]. Recall that minimizing the upper bound $M_{\gamma}$ of the induced- $\mathcal{L}_{2}$ norm from $d$ to $e$ is not in convex optimization, since $\lambda_{1}, \lambda_{2}$, and $\kappa$ are highly related with $\gamma$ in Eq. (23). Using line searching over the $\lambda_{1}, \lambda_{2}$, and $\kappa$ ranges, the analysis results are calculated in Table 2 . When $T_{o_{3}}$ is fixed as $0.2 \mathrm{sec}$, the upper bound of the induced- $\mathcal{L}_{2}$ norm is calculated as 0.68 for $K_{A}$ and 2.87 for $K_{B}$ with constant matrix $P$ and 0.54 for $K_{A}$ and 0.84 for $K_{B}$ with parameter dependent matrix $P(\bar{\rho})$.

It is noticed from Eq. (31) that the time constants $T_{o_{i}}$ are not related with optimized $\lambda_{1}, \lambda_{2}, \kappa$, and $\gamma$. Thus, with fixed $\kappa, \lambda_{1}, \lambda_{2}$, and $\gamma$ values for each controller, the $M_{\gamma}$ variations are calculated due to $T_{o_{3}}$ changes and are shown in Figure 5. It is observed from Figure 5, that performance analysis results are significantly different using constant matrix $P$ and a parameter dependent matrix $P(\bar{\rho})$. Based on performance analysis result with a parameter dependent matrix $P(\bar{\rho})$, it is easily noticed that when a false fault detection time is short $\left(T_{o_{3}}<0.22 \mathrm{sec}\right)$, the $M_{\gamma}$ with the $K_{A}$ controller is less than that with the $K_{B}$ controller. When a false fault detection time is long $\left(T_{o_{3}}>0.22 \mathrm{sec}\right)$, the $M_{\gamma}$ values are vise versa. The analysis results imply that $K_{A}$ controller leads to less performance degradation for the $T_{o_{3}}<0.22 \mathrm{sec}$ case and $K_{B}$ controller leads to less performance degradation for the $T_{o_{3}}>0.22 \mathrm{sec}$ case. 
Recall that $M_{\gamma}$ in this FTC system analysis represents the upper bound of the induced norm of $\left\|\theta-\theta_{\text {ideal }}\right\|_{2}$ from a given command $\|d\|_{2}$. In order to validate the FTC system performance analysis results, the closed-loop system is simulated with assumption of false fault detection $T_{o_{3}}=0.1,0.2,0.4$ and $0.8 \mathrm{sec}$ for each controller, respectively.

In Figure 6, the error between pitch angle responses and ideal responses $\theta_{\text {ideal }}$ are plotted for each case. It is obviously noticed that the error norm of $\left\|\theta-\theta_{\text {ideal }}\right\|_{2}$ with the $K_{A}$ controller is larger than that with the $K_{B}$ controller at the $T_{3}=0.4$ and $0.8 \mathrm{sec}$ cases. Also, the error norm with the $K_{B}$ controller is larger than that with the $K_{A}$ controller at the $T_{o_{3}}=0.1$ and $0.2 \mathrm{sec}$ cases. The parameter dependent analysis results in Figure 5 correspond to the simulation results in Figure 6. Note that using this analysis tool, we can calculate the upper bound variation of the induced- $\mathcal{L}_{2}$ norm due to $T_{0_{2}}$ variation which represents parameter transient time. In this example, $\lambda_{1}$ and $\lambda_{2}$ are too small to see effects of $T_{0_{2}}$ variations.

\section{CONCLUSION}

In this paper, the FTC system analysis problem is formulated into the optimization problem subject to LMI constraints which are evaluated at grid points over the stable/unstable parameter subspaces. From the stability analysis, the tolerance of false fault detection which causes local instability can be calculated for a FTC system to achieve exponential stability. From the performance analysis, the upper bound of the induced- $\mathcal{L}_{2}$ norm of the FTC system represents worst-case performance during the detection time intervals of FDI modules. The norm bound is calculated as a function of detection time interval and exponential decay rates over each parameter subspace. It indicates performance degradation due to parameter transients during the interval in which the closed-loop system may be locally unstable. In this paper, the usage of the FTC system analysis is demonstrated via the analysis of the FTC HiMAT system.

\section{ACKNOWLEDGMENTS}

The technical monitor for this work was Dr. Christine Belcastro at NASA Langley Research Center.

\section{REFERENCES}

[1] Belcastro, Christine and Belcastro, Celeste, "Application of failure detection, identification, and accommodation methods for improved aircraft safety," in Proceedings of American Control Conference, 2001, pp. 2623-2624, American Automatic Control Council, Evanston, IL.

[2] Ly, U. and Ho J.K., "Fault Tolerant Control Laws," NASA Langley Research Center, NASA CR - 178094, 1986. Nation Aeronautics and Space Administration.

[3] Chen, J., Patton, R.J., and Chen, Z, "An LMI approach to Fault-Tolerant Control of Uncertain System," in Proceeding of the 1998 International Symposium on Intelligent 
Control, 1998, pp. 175-180, Institute of Electrical and Electronics Engineers, Piscataway, N.J.

[4] Ganguli, S., Marcos, A., and Balas, G., "Reconfigurable LPV Control Design for Boeing 747-100/200 Longitudinal Axis," in Proceedings of American Control Conference, (Anchorage, AK), 2002, pp. 3612-3617, American Control Conference Council, Evanston, IL.

[5] Shin, J-Y., Wu, N.E., and Belcastro, C., "Linear Parameter Varying Control Synthesis for Actuator Failure, Based on Estimated Parameter," in AIAA Guidance, Navigation and Control, No. AIAA-2002-4546, 2002, American Institute of Aeronautics and Astronautics.

[6] Wu, N. Eva, Zhang, Y., and Zhou, K., "Detection, Estimation, and Accommodation of Loss of Control Effectiveness," International Journal of Adaptive Control and Signal Processing, Vol. 14, No. 7, 2000, pp. 775-795.

[7] Szaszi, I., Marcos, A., Balas, G., and Bokor, J., "LPV Detection Filter Design for Boeing 747-100/200," in AIAA Guidance, Navigation and Control, No. AIAA 2002-4957, 2002, American Institute of Aeronautics and Astronautics.

[8] Hespanha, J., Yakimenko, O., Kaminer, I., and Pascoal, A., "LPV Systems with Brief Instabilities:Application to Integrated Vision/IMU Navigation," in IEEE Proceeding of the Conference on Decision and Control, 2001.

[9] Shin, J.-Y., Worst-Case Analysis and Linear Parameter-Varying Gain-Scheduled Control of Aerospace Systems. PhD thesis, Department of Aerospace Engineering and Mechanics, University of Minnesota, 2000.

[10] Wu, F., Control of Linear Parameter Varying Systems. PhD thesis, Department of Mechanical Engineering, University of California, Berkeley, 1995.

[11] Shin, J-Y. and Belcastro, C., "Analysis of A Fault Tolerant Control System: False Fault Detection Case," in 5th IFAC Symposium on Fault Detection, Supervision and Safety of Technical Processes, June 2003.

[12] Balas, G., Doyle, J., Glover, K., and Packard, A., $\mu$ Analysis and Synthesis Toolbox. Natick, MA: The Mathworks, Inc., 1995.

[13] Keller, J. K. and Darouach, M., "Optimal two-stage Kalman filter in the presence of random bias," Automatica, Vol. 33, No. 9, 1997, pp. 1745-1748. 


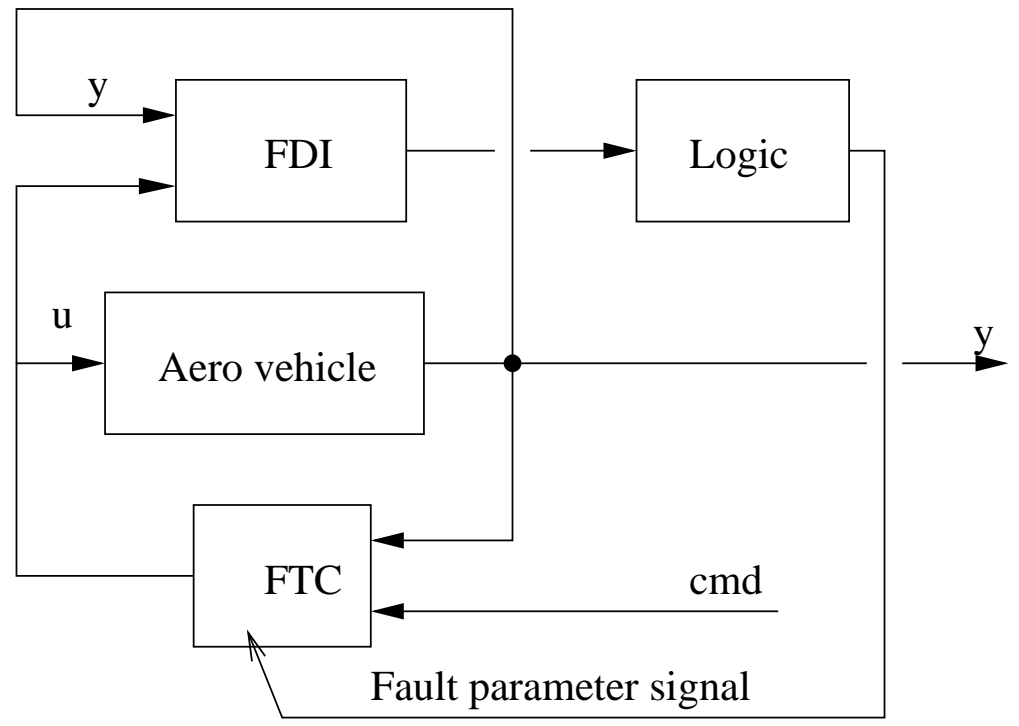

Figure 1: Structure of a fault tolerant control system. 


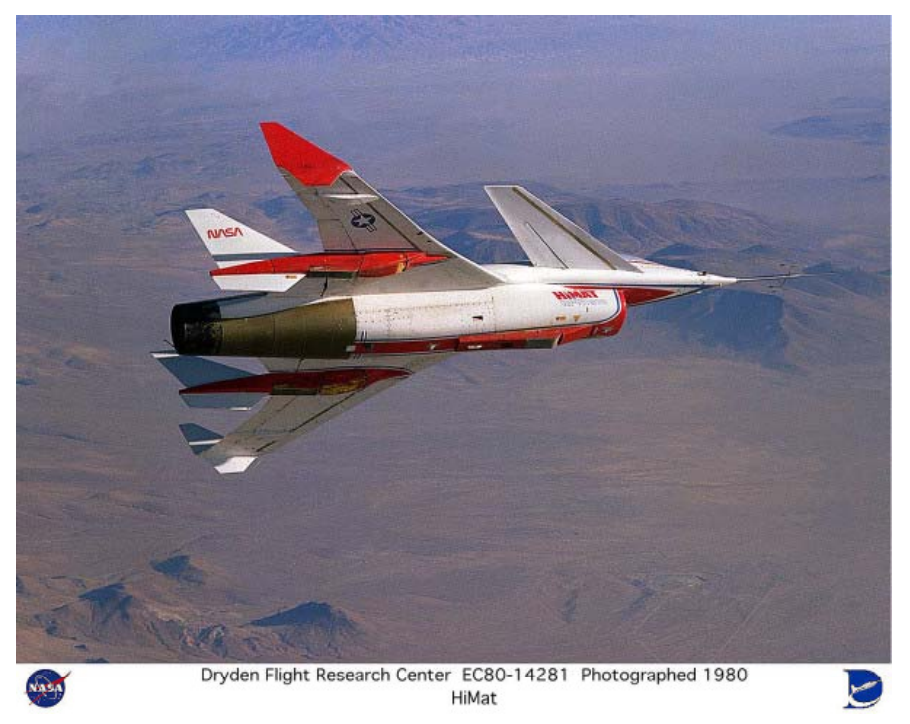

Figure 2: HiMAT vehicle. 

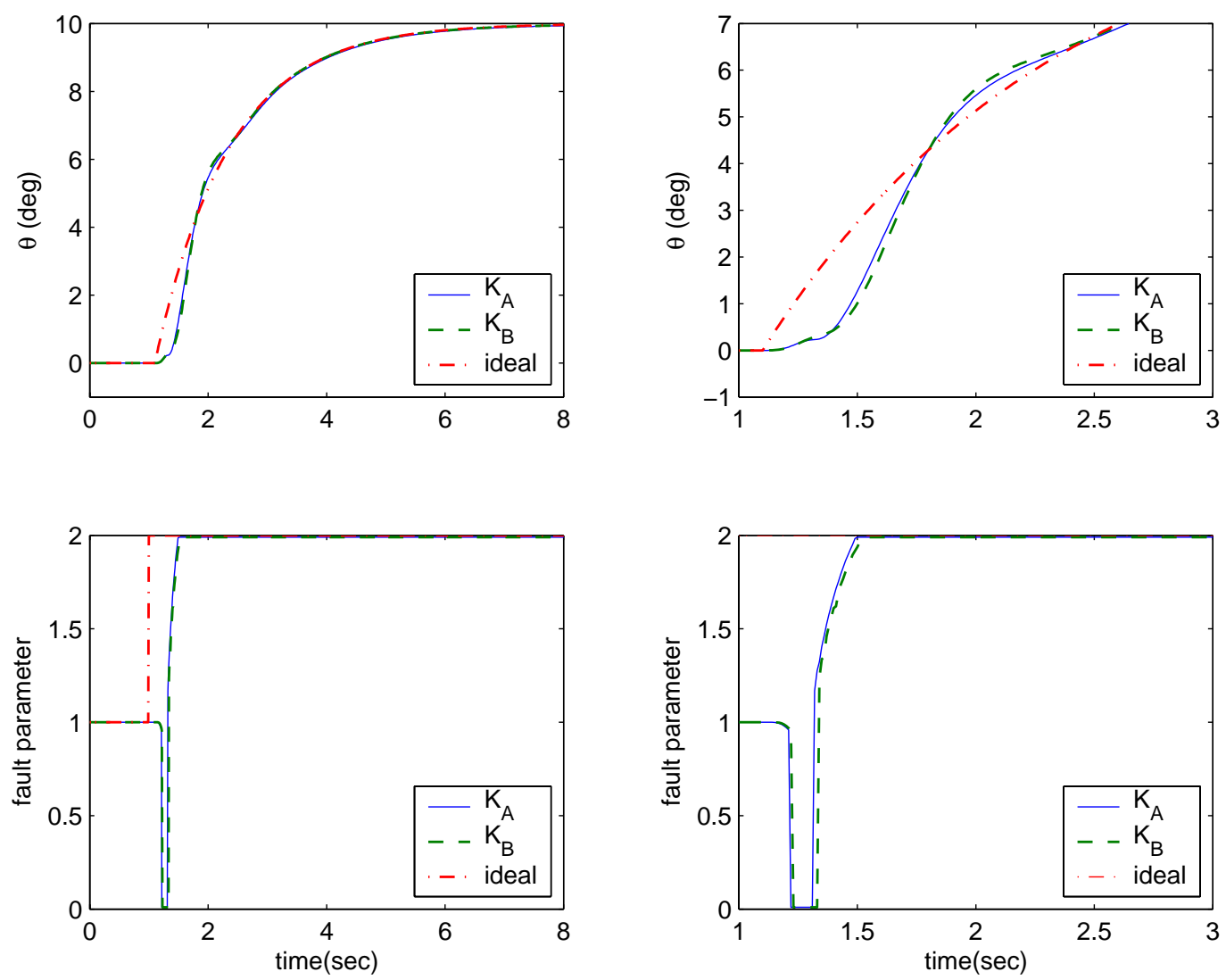

Figure 3: Nonlinear simulations of a FTC HiMAT Vehicle. 


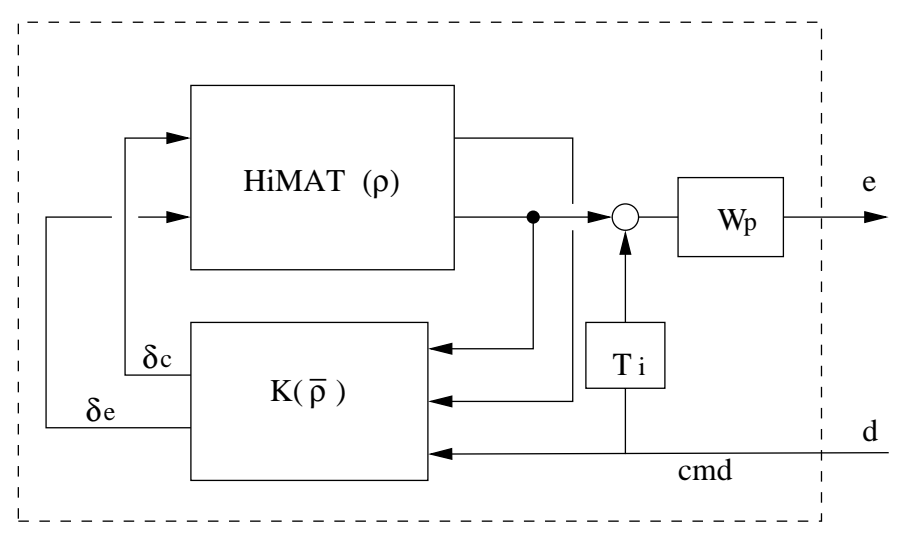

Figure 4: The weighted closed-loop system of a HiMAT vehicle 

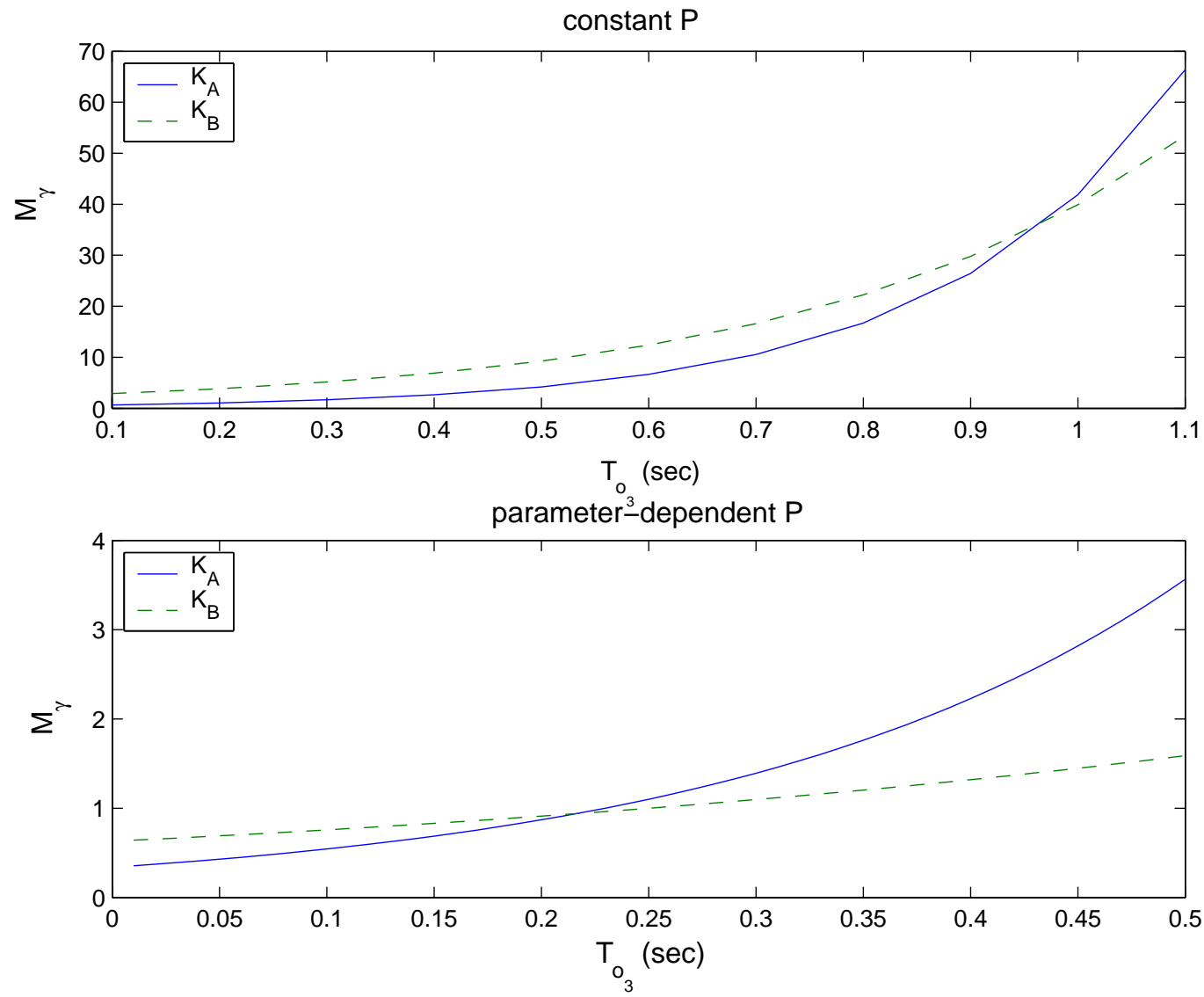

Figure 5: Variations of $M_{\gamma}$ due to $T_{o_{3}}$ changes. 

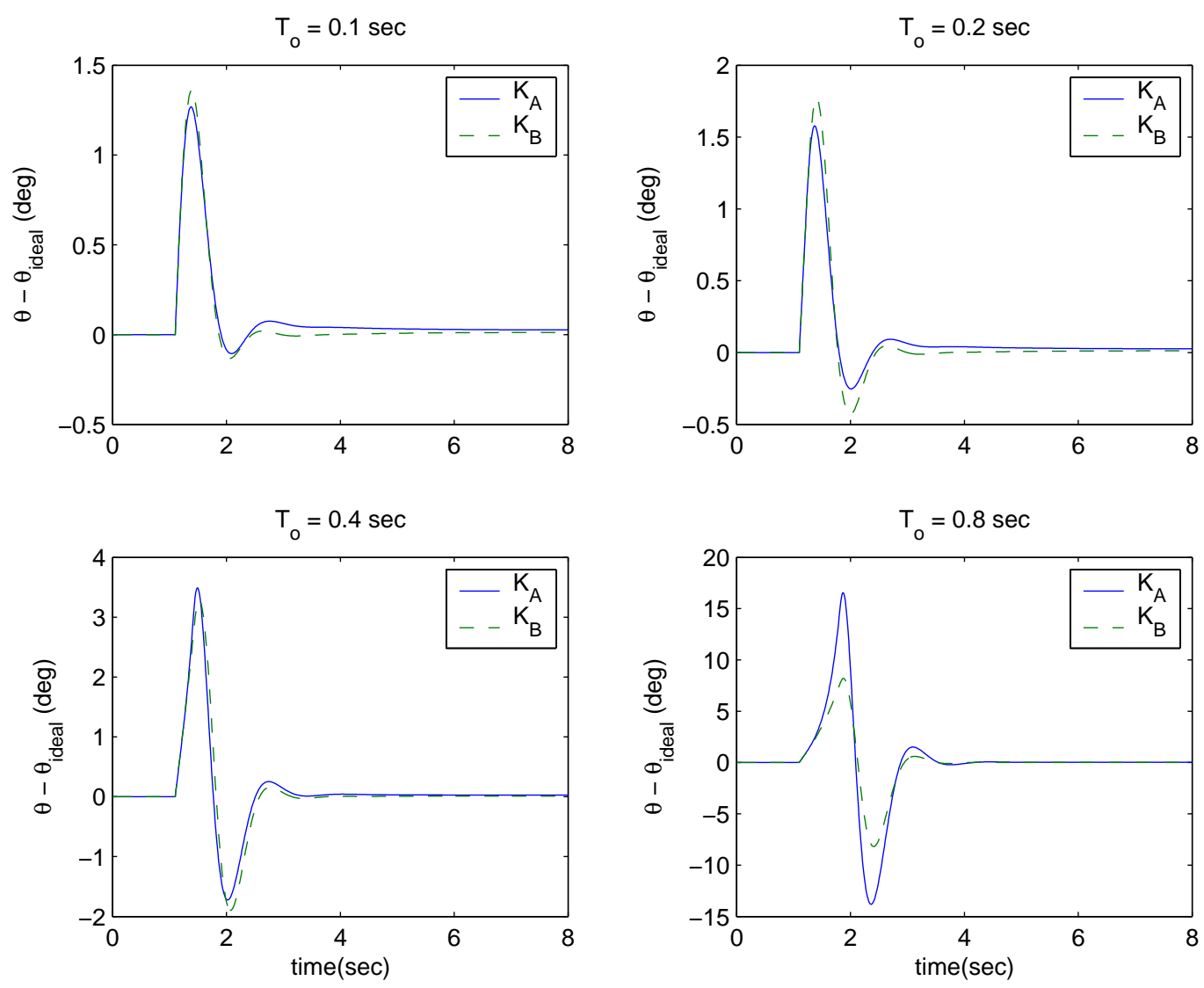

Figure 6: Simulation results with different $T_{o_{3}}$ values: $0.1,0.2,0.4$ and 0.8 sec. 
The public reporting burden for this collection of information is estimated to average 1 hour per response, including the time for reviewing instructions, searching existing

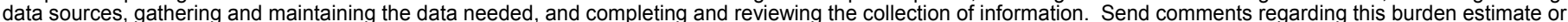

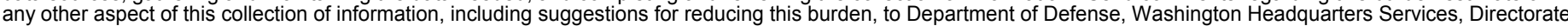

for Information Operations and Reports (0704-0188), 1215 Jefferson Davis Highway, Suite 1204, Arlington, VA 22202-4302. Respondents should be aware that

notwithstanding any other provision of law, no person shall be subject to any penalty for failing to comply with a collection of information if it does not display a currently

valid OMB control number.

PLEASE DO NOT RETURN YOUR FORM TO THE ABOVE ADDRESS

1. REPORT DATE (DD-MM-YYYY) 1 2. REPORT TYPE 3. DATES COVERED (FrOm - To)

$01 / 12 / 2003$

\section{TITLE AND SUBTITLE}

Parameter Transient Behavior Analysis on Fault Tolerant Control System

\section{6. $\operatorname{AUTHOR(S)}$}

Shin, Jong-Yeob

\section{5a. CONTRACT NUMBER}

5b. GRANT NUMBER

NCC-1-02043

5c. PROGRAM ELEMENT NUMBER

5d. PROJECT NUMBER

\section{5e. TASK NUMBER}

5f. WORK UNIT NUMBER

762-60-31-02-00

\section{PERFORMING ORGANIZATION NAME(S) AND ADDRESS(ES)}

NASA Langley Research Center

Hampton, VA 23681-2199

National Institute of Aerospace (NIA)

144 Research Drive

Hampton, VA 23666
8. PERFORMING ORGANIZATION REPORT NUMBER

NIA Report No. 2003-05

10. SPONSORING/MONITOR'S ACRONYM(S)

NASA

11. SPONSORING/MONITORING REPORT NUMBER

NASA/CR-2003-212682

\section{DISTRIBUTION/AVAILABILITY STATEMENT}

Unclassified - Unlimited

Subject Category 64

Availability: NASA CASI (301) 621-0390 Distribution: Nonstandard

\section{SUPPLEMENTARY NOTES}

Langley Technical Monitor: Christine Belcastro

\section{ABSTRACT}

In a fault tolerant control (FTC) system, a parameter varying FTC law is reconfigured based on fault parameters estimated by fault detection and isolation (FDI) modules. FDI modules require some time to detect fault occurrences in aero-vehicle dynamics. This paper illustrates analysis of a FTC system based on estimated fault parameter transient behavior which may include false fault detections during a short time interval. Using Lyapunov function analysis, the upper bound of an induced-L_2 norm of the FTC system performance is calculated as a function of a fault detection time and the exponential decay rate of the Lyapunov function.

\section{SUBJECT TERMS}

Fault Tolerant Control System; LPV Control System

\begin{tabular}{|c|c|c|c|c|c|}
\hline \multicolumn{3}{|c|}{ 16. SECURITY CLASSIFICATION OF: } & \multirow{3}{*}{$\begin{array}{l}\text { 17. LIMITATION OF } \\
\text { ABSTRACT } \\
\text { UU }\end{array}$} & \multirow{3}{*}{$\begin{array}{l}\text { 18. NUMBER } \\
\text { OF } \\
\text { PAGES } \\
24\end{array}$} & \multirow{2}{*}{$\begin{array}{l}\text { 19b. NAME OF RESPONSIBLE PERSON } \\
\text { STI Help Desk (email: help@ @ sti.nasa.gov) }\end{array}$} \\
\hline a. REPORT & b. ABSTRACT & c. THIS PAGE & & & \\
\hline $\mathrm{U}$ & $\mathrm{U}$ & $\mathrm{U}$ & & & $\begin{array}{l}\text { 19b. TELEPHONE NUMBER (Include area code) } \\
\text { (301) 621-0390 }\end{array}$ \\
\hline
\end{tabular}

